\title{
Statistical characteristics of medium-scale traveling ionospheric disturbances revealed from the Hokkaido East and Ekaterinburg $\mathrm{HF}$ radar data
}

\author{
Alexey V. Oinats ${ }^{1 *}$, Nozomu Nishitani ${ }^{2}$, Pavlo Ponomarenko ${ }^{3,4}$, Oleg I. Berngardt ${ }^{1}$ and Konstantin G. Ratovsky ${ }^{1}$
}

\begin{abstract}
We present a statistical study of medium-scale traveling ionospheric disturbances (MSTIDs) using the Hokkaido East $\left(43.53^{\circ} \mathrm{N}, 143.61^{\circ} \mathrm{E}\right)$ and Ekaterinburg $\left(56.42^{\circ} \mathrm{N}, 58.53^{\circ} \mathrm{E}\right)$ high-frequency (HF) radar data. Radar datasets are available from 2007 to 2014 for the Hokkaido and from 2013 to 2014 for the Ekaterinburg radar. In the case of the Hokkaido East radar, we have utilized the elevation angle information to study the MSTIDs propagating at the heights of the $E$ and $F$ ionospheric regions separately. We have analyzed the diurnal and seasonal behavior of the following medium-scale traveling ionospheric disturbance (MSTID) parameters: propagation direction, apparent horizontal velocity and wavelength, period, and relative amplitude. The F region MSTID azimuthal patterns were observed to be quite similar by the two radars. The E region northwestward MSTIDs (from $280^{\circ}$ to $320^{\circ}$ ) were typical of summer daytime. Comparison with the horizontal wind model (HWMO7) has showed that the dominant MSTID propagation directions match the anti-wind direction well, at least during sunlight hours. We have also found that the wavelength and period tend to decrease with an increase in solar activity. On the contrary, the relative amplitude increases with an increase in solar activity. Moreover, the relative amplitude tends to increase with increasing auroral electrojet (AE) index, as do the wavelength and velocity.
\end{abstract}

Keywords: Medium-scale traveling ionospheric disturbances, Atmospheric gravity waves, High-frequency radar, Hokkaido East SuperDARN radar, Ekaterinburg HF radar

\section{Introduction}

Medium-scale traveling ionospheric disturbances (MSTIDs) are wave-like electron density disturbances with horizontal wavelengths of about several hundreds of kilometers and periods of 15 min up to $1 \mathrm{~h}$ (Hunsucker 1982; Hocke and Schlegel 1996). The daytime mid-latitude MSTIDs are assumed to be caused by atmospheric gravity waves (AGWs), propagating at ionospheric heights. The nighttime midlatitude MSTIDs are likely to be associated with Perkins instability (Shiokawa et al. 2003b; Otsuka et al. 2004; Tsunoda 2006).

In the last 15 years, several papers have been published that focus on the statistical studies of MSTIDs using different techniques and observational facilities (Afraimovich

\footnotetext{
*Correspondence: oinats@iszf.irk.ru

'Institute of Solar-Terrestrial Physics SB RAS, 664033, Lermontov St., 126a, P.O. Box 291, Irkutsk, Russia

Full list of author information is available at the end of the article
}

et al. 1999, 2000; Lastovicka 2001; Shiokawa et al. 2003a, 2009; He et al. 2004; Kotake et al. 2007; Ishida et al. 2008; Klausner et al. 2009; Fukushima et al. 2012; Otsuka et al. 2011, 2013; Grocott et al. 2013; Medvedev et al. 2013; Ichihara et al. 2013; Frissell et al. 2014). Some of these studies have used high-frequency (HF) ground backscatter (GB) data (He et al. 2004; Ishida et al. 2008; Grocott et al. 2013; Ichihara et al. 2013; Frissell et al. 2014). The possibility of studying MSTIDs/AGWs using HF radar GB data was first proposed by Samson et al. (1990), who showed that MSTIDs manifest themselves as periodic variations of the GB power. One of the GB characteristics, which is measured by HF radar and is sensitive to MSTIDs, is the minimum slant range corresponding to the skip distance (Arnold et al. 1998; Stocker et al. 2000; Karhunen et al. 2006).

In our previous paper (Oinats et al. 2015), we described an automated technique that uses a cross-correlation 
analysis of minimum slant range variations to determine the traveling ionospheric disturbance (TID) parameters. We presented diurnal and seasonal behavior of the MSTID azimuth and apparent horizontal velocity as revealed from the 2011 Hokkaido East super dual auroral radar network (SuperDARN) data. Here, we present a more comprehensive analysis that includes the occurrence rates for the MSTID azimuth, horizontal phase velocity, period, horizontal wavelength, and relative amplitude calculated using the multi-year datasets of two mid-latitude HF radars: Hokkaido East and Ekaterinburg. The Hokkaido East radar dataset covers an 8-year period, from 2007 to 2014. The Ekaterinburg radar dataset has been processed from 2013 to 2014. As compared to our previous study, we have significantly improved a preprocessing algorithm for slant range variation extraction and mapping using calibrated elevation angles and range offset information for the Hokkaido East radar (Ponomarenko et al. 2015). This allowed us to study the disturbances propagating in the $\mathrm{E}$ and $\mathrm{F}$ regions of the ionosphere separately. Comparison of the MSTID characteristics obtained from measurements by two radars and for two ionospheric regions could provide important information about the regional (latitudinal and altitudinal) characteristics of the AGWs. Using the extended radar datasets, we have studied the solar cycle and geomagnetic activity dependences of MSTID statistical characteristics and have compared the results with the horizontal wind model (Drob et al. 2008) to check the AGW filtering properties of neutral winds.

\section{Instrumentation}

The two mid-latitude HF radars, the Hokkaido East $\left(43.53^{\circ} \mathrm{N}, 143.61^{\circ} \mathrm{E}\right)$ and Ekaterinburg $\left(56.42^{\circ} \mathrm{N}\right.$, $58.53^{\circ} \mathrm{E}$ ), are very similar. There are 16 azimuths, called "beams," that form an approximate $50^{\circ}$-width field-of-view (FOV). Radars provide measurement of range-time dependencies of several echo parameters, such as power, Doppler velocity, Doppler spectral width, and elevation angle at each beam with a temporal resolution of 1-2 min. Figure 1 is a map of the radar locations and their FOVs. The only difference between the two radars, which is important for further consideration, is that the Ekaterinburg radar has no interferometer phased-array and it does not measure the elevation angle. Elevation angles are necessary for mapping the radar data and more accurate determination of the effective height where the HF waves are reflected. In case of the Hokkaido East radar, elevation angle measurements have been calibrated in 2014 (Ponomarenko et al. 2015), and it allowed us to separate $\mathrm{E}$ and $\mathrm{F}$ region echoes.

\section{Data processing}

The main idea of our technique is to fit the TID analytical model to minimum slant range variations observed on different radar beams (Oinats et al. 2013). The steps of data processing and analysis are almost the same as described in Oinats et al. (2015). Here, we list them briefly and emphasize the differences between present and previous techniques.

In our analysis, we have used the GB echoes identified using the standard technique (Blanchard et al. 2009). We have excluded the echoes originating behind the radar by interferometer data analysis (Milan et al. 1997). The technique for identification of such echoes is similar to those described in Bland et al. (2014). For reliable identification of one-hop GB echoes, we have considered a number of criteria for the slant range, effective reflection height, and power for a certain beam, local time, and date. More specifically, we have developed a GB
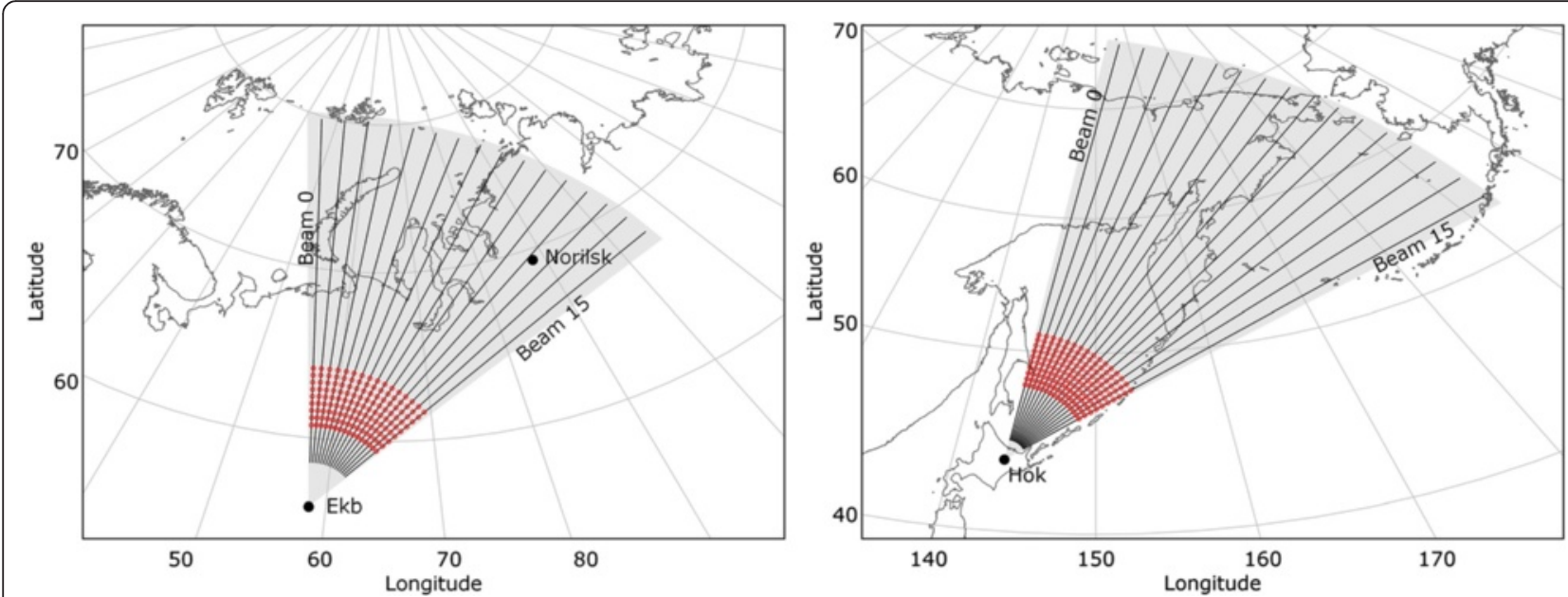

Fig. 1 Location of the Ekaterinburg and Hokkaido East HF radars and their FOV. Approximate location of the ionospheric reflection points is shown by red circles 
model (GBM) for each radar data (Oinats et al. 2016). GBM is based on HF propagation simulation in the framework of the waveguide approach (Oinats et al. 2012) and IRI-2012 ionosphere (Bilitza et al. 2014). It includes a set of the following HF GB characteristics: the slant range corresponding to the skip distance, elevation angle, true height of reflection, slant range, and ground distance to the ionospheric reflection region. All the characteristics have been calculated for each radar beam for the entire period under consideration. Using the model, we have determined possible slant ranges and effective heights for each HF channel. This makes it possible to separate the regular GB reflected by E and F2 ionospheric layers from other echoes. Since all the radar range gates satisfying the mask are determined at a certain local time, we searched for the range gate, that received the maximum power, and recorded the corresponding slant range and elevation angle values.

We determined the ground distance, $D_{\text {ref }}$, from the radar site to the ground projection of reflection region, assuming that the Breit and Tuve's theorem (Davies 1990) holds for the spherically symmetric ionosphere

$$
D_{r e f}=a \cdot \operatorname{arctg}\left(\frac{r \cos \Delta}{g r \cdot a+r \sin \Delta}\right),
$$

where $a$ is the Earth's radius, $r$ and $\Delta$ are the slant range and elevation angle, respectively. Regular gradients are compensated in Eq. (1) by the gradient factor, $g r$, which is taken from the GBM for a certain beam, local time, and date. The appropriate latitude and longitude are calculated with reference to the beam azimuth and radar coordinates. In case of absence of measured elevation angle, i.e., for the Ekaterinburg radar, we first calculated the effective elevation angle from GBM and then determined corresponding ground distance using Eq. (1).

The obtained slant range-time dependencies are filtered by finite impulse response (FIR) band-pass filter, which passes the periods from $10 \mathrm{~min}$ to $2 \mathrm{~h}$ to remove the diurnal trend. Filtered dependencies are fitted by penalized regression splines (Ruppert et al. 2003). Finally, we recalculated the dependencies on an equidistant time grid common for all beams.

In the next step, we calculated the cross-correlation functions between the dependencies for all possible beam pairs at each moment. Statistical sampling size is fixed and corresponds to a time interval of $6 \mathrm{~h}$. Maximum cross-correlation functions correspond to the observed time lags, $\Delta t$, between the dependencies. In addition, we determined the prevailing period and root mean square (RMS) of deviations for each variation. Simulation provided by Stocker et al. 2000 and Oinats et al. 2012 showed that the RMS of slant range deviation is nearly proportional to the amplitude of electron density perturbation (at least for amplitudes less than $\sim 20 \%$ ). Therefore, it could be considered as some estimation of the TID amplitude. After that, we constructed a two-dimensional squared deviation between observed and theoretical time lags

$$
\delta(\Phi, v)=\sum_{j, i=0, j \neq i}^{15}\left(\Delta t_{j i}^{\prime}-\Delta t_{j i}(\Phi, v)\right)^{2},
$$

where $\Phi$ and $v$ are the TID azimuth (clockwise from the north) and horizontal phase velocity, respectively; $\Delta t_{j i}^{\prime}$ is the theoretical time lag; $i$ and $j$ are the beam numbers. We assume that TID has plane phase front within the radar FOV and vertical component of the phase velocity is equal to zero. Under these assumptions, the theoretical time lag between two reflection regions on different beams is given by

$$
\begin{aligned}
\Delta t_{j i}(\Phi, v)= & \frac{a}{v}\left[\left(\left(\phi_{j}-\phi^{\prime}\right) \cos \theta_{j}-\left(\phi_{i}-\phi^{\prime}\right) \cos \theta_{i}\right)\right. \\
& \left.\sin \Phi+\left(\theta_{j}-\theta_{i}\right) \cos \Phi\right]
\end{aligned}
$$

where $\left(\theta_{i}, \phi_{i}\right)$ and $\left(\theta_{j}, \phi_{j}\right)$ are the coordinates (latitude and longitude) of the reflection regions for $i$-th and $j$-th beam numbers, and $\left(\theta^{\prime}, \phi^{\prime}\right)$ are the latitude and longitude of the radar. In Eq. (2), we take into account only those time lags for which the correlation coefficient exceeds the threshold of 0.85 . Minimization of the deviation is performed using the Levenberg-Marquardt algorithm for optimizing the parameters of non-linear regression models. The successful minimization gives us appropriate MSTID azimuth and horizontal velocity values at a certain local time.

In the final step, we determined other MSTID parameters by averaging over beams. We took into account only those beams that were used in minimization. Period is calculated as a median of a set of prevailing periods. Horizontal wavelength is defined from the calculated period and velocity. The amplitude is expressed as an average of the deviation RMS. Relative amplitude is equal to the ratio between the amplitude and average slant range.

The above processing was carried out for each local time and date. As a result, we obtained the diurnal dependencies of five MSTID characteristics for each date under the period of consideration.

\section{Results and discussion}

\section{Diurnal and seasonal dependence of MSTID azimuth occurrence rate}

Figure 2 shows the diurnal variations of occurrence rate of the MSTID propagation direction for the entire period and three seasons: equinox (March, April, September, and 
October), summer (May, June, July, and August) and winter (November, December, January, and February). We should note that the regular GB echo is not observed for all local times. For example, there is no GB data at all during nighttime for the $E$ region due to its low critical frequency. The GB data for the $F$ region during nighttime in winter months is also insufficient to form a significant distribution (this explains some noisiness of the winter MSTID distributions). Therefore, the occurrence rate hereinafter is determined as a ratio between the observation duration of a given value of the MSTID parameter and the general observation interval at a certain local time. Figure $2 \mathrm{a}, \mathrm{b}$ shows the color-coded occurrence rate variations corresponding to the heights of the Hokkaido $\mathrm{E}$ and F2 regions (Hok-E and Hok-F), respectively. The occurrence rate is calculated in each azimuth bin of $10^{\circ}$ width (vertical axis) and for each hour (local time, horizontal axis). In Fig. 2b, one can see four dominant directions with enhanced occurrence: morning northeastward (from $20^{\circ}$ to $50^{\circ}$ at 4-6 local time [LT]), daytime southeastward (from $100^{\circ}$ to $140^{\circ}$ at 7-15 LT), nighttime and evening southwestward (from $190^{\circ}$ to $220^{\circ}$ at $15-18 \mathrm{LT}$ and $21-3$

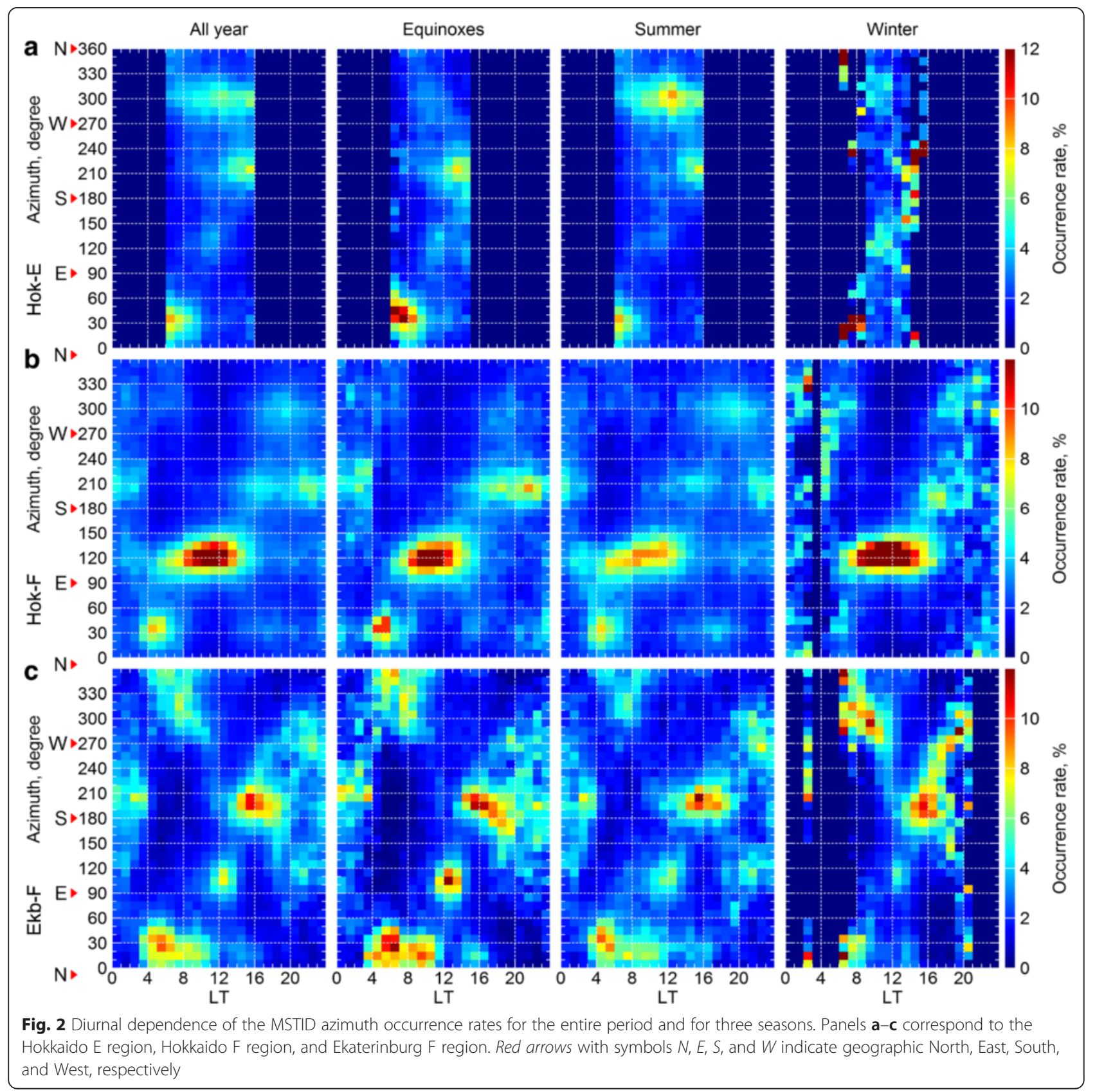


LT), and evening northwestward (from $280^{\circ}$ to $310^{\circ}$ at 18 $21 \mathrm{LT})$. The directions vary seasonally. The maximum occurrence rate is for the daytime southeastward disturbances, which are most prominent during winter. Morning northeastward disturbances are most pronounced during equinox. Nighttime southwestward disturbances are most pronounced in equinox-summer. In Oinats et al. (2015), we have already discussed these four directions for the 2011 Hokkaido data. We have indicated a good agreement between them and results of many other researchers (Afraimovich et al. 1999; Shiokawa et al. 2003a; Kotake et al. 2007; Ishida et al. 2008; Otsuka et al. 2011; Medvedev et al. 2013), excluding the northwestward disturbances that we previously found to be typical of the summer daytime (see Fig. $6 \mathrm{c}$ in Oinats et al. (2015)). Comparing Fig. 2a, b, one can see that northwestward summer daytime MSTIDs actually propagate at heights of the E region (see azimuths from $280^{\circ}$ to $320^{\circ}$ at $8-16$ LT in Fig. 2a). Thus, not distinguishing $\mathrm{E}$ and $\mathrm{F}$ region echoes explains our previous result, which was obtained without their separation. In Fig. 2a, there are two other dominant directions in Hok-E: morning northeastward (from $20^{\circ}$ to $50^{\circ}$ at 6-9 LT) and evening southwestward (from $190^{\circ}$ to $215^{\circ}$ at $13-16 \mathrm{LT}$ ). These two directions look very similar to the corresponding Hok-F directions, but they are observed slightly later or earlier accordingly. The possibility of the southwestward or northwestward MSTIDs in the E region was reported by Koustov et al. (2013), who had also used the Hokkaido radar data in their study. The southeast daytime peak is significantly weaker for Hok-E as compared to Hok-F (see azimuths $\sim 120^{\circ}$ at $\sim 12$ LT in Fig. 2a).

Figure 2c shows the occurrence rates calculated for the F2 region from the Ekaterinburg data (Ekb-F). Comparing with Fig. 2 b, one can see that the occurrence patterns for the two radars are very close to each other. However, there is a difference in the absolute values of the occurrence rate. For example, the most prominent Hok-F southeast daytime peak is rather weak for the Ekb-F. In addition, the peaks for the Ekaterinburg radar are shifted slightly to greater local time. The shift can be partially explained by the fact that the registration region of the Hokkaido East radar is located mainly to the east from the radar site in a different time zone $(+1 \mathrm{~h})$.

One of the prominent effects of the relation of MSTID with $\mathrm{AGW}$ is the filtering by neutral winds. According to the theoretical concept, if neutral wind has a component parallel to the wave vector, it causes a Doppler shift of GW frequency (Waldock and Jones 1984). Depending on the mutual orientation of the wave, wind, and their parameters, critical coupling or reflection may occur. Therefore, not all GWs originating from the lower atmosphere (troposphere) and initially having all possible directions can reach the ionosphere. This results in the appearance of a certain diurnal pattern of the observed MSTID azimuths. Many researchers have reported observational evidences of this effect (Kalikhman 1980; Waldock and Jones 1986; Afraimovich et al. 1999; Kotake et al. 2007). Kalikhman (1980) has presented a statistical study based on Doppler and arrival angle data obtained by the F2 layer vertical sounding from 1975 to 1976 at Irkutsk. He showed that the direction of MSTID motion during daytime agrees well with the thermospheric wind direction and is oriented opposite to it. Waldock and Jones (1986) observed the MSTIDs from 1972 to 1975 at Leicester by means of an HF Doppler technique and found that the wave azimuth rotates clockwise through $360^{\circ}$ in $24 \mathrm{~h}$. However, the direction of disturbance motion is displaced from the anti-wind direction and is located at an azimuth of $130^{\circ}-140^{\circ}$ relative to the wind. Afraimovich et al. (1999) analyzed the annual data from the transionospheric radio interferometer that measured the parameters of the radio signal at $136 \mathrm{MHz}$ from the ETS 2 geostationary satellite. They found that the mean daytime MSTID direction coincides with the anti-wind direction with a RMS of about $15^{\circ}$ for the autumn-winter season. However, they did not find evidence of clockwise rotation of MSTID direction for the spring-summer season. Kotake et al. (2007) presented a study based on the analysis of the twodimensional maps of total electron content (TEC) perturbations constructed from the GPS network data in Southern California in 2002. They found that the propagation direction of daytime MSTIDs tends to rotate clockwise from $90^{\circ}$ to $240^{\circ}\left(120^{\circ}\right.$ to $\left.240^{\circ}\right)$ in azimuth between 6 and 15 LT in equinox (winter). Similar results were also obtained by Otsuka et al. (2011) from the GPS network observations in Japan in 2002.

To check the filtering effect of the neutral wind, we calculated the relative occurrence rate for the neutral wind direction using the horizontal wind model (HWM07) (Drob et al. 2008). The wind azimuth is calculated for each date and local time from the observational period when a regular GB echo is predicted by the corresponding GBM for the central beam of radar (beam \#8). The height and coordinates in the calculation are the true reflection height and reflection point coordinates, respectively, taken from the GBM for the corresponding HF channel. Figure $3 \mathrm{a}-\mathrm{c}$ shows the occurrence rates for Hok-E, Hok-F, and Ekb-F, respectively, by the gray color according to the color bars to the right of the plots. Since the Ekaterinburg radar did not provide the elevation angle, we used the combined Ekaterinburg GBM for the $E$ and $F$ channels $(E+F)$. Similar to Fig. 2, the occurrence rate is determined as a ratio between the predicted duration of a given wind azimuth and the general interval of GBM prediction at a certain local time. Vertical and horizontal axes show 


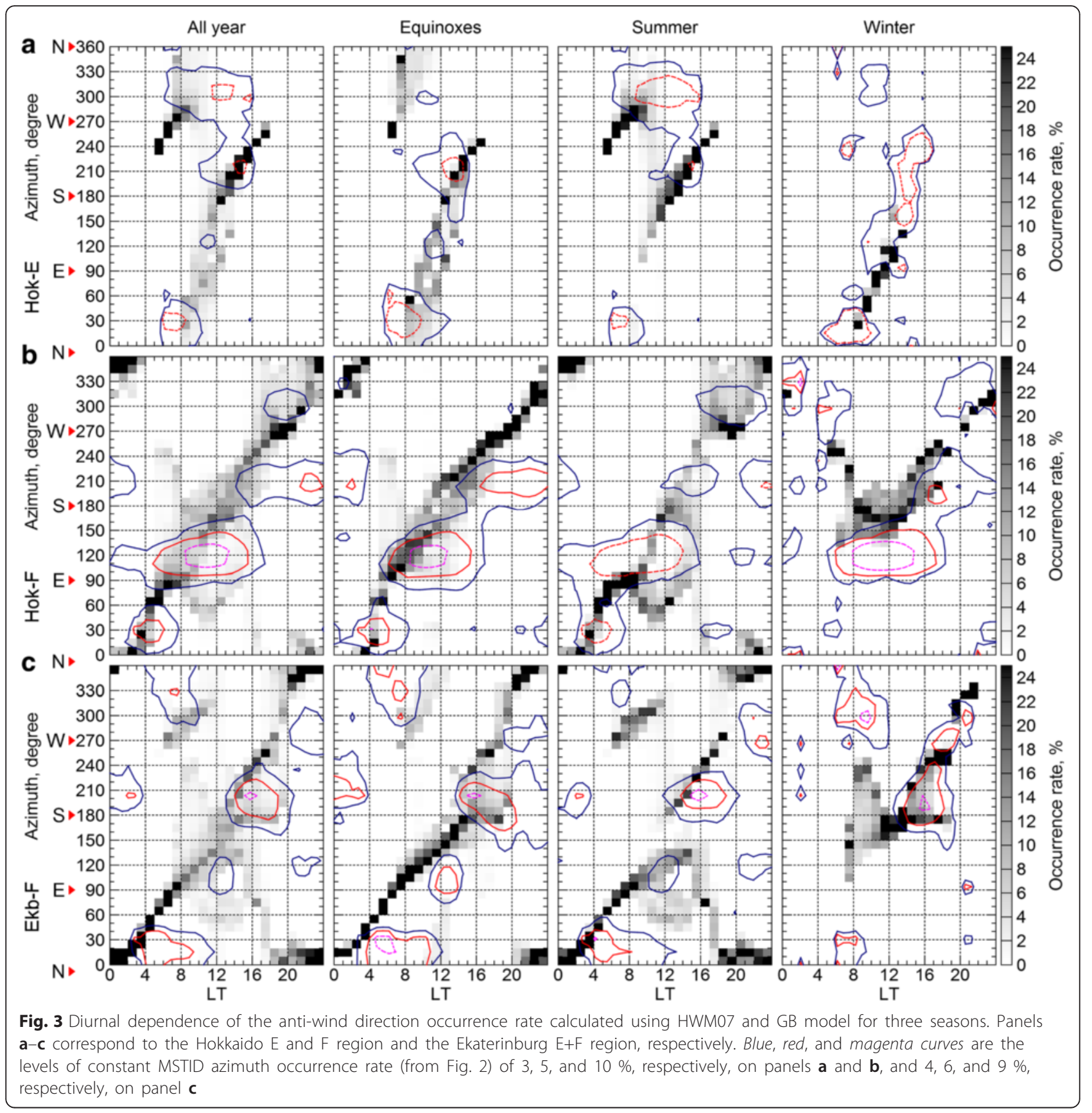

the anti-wind azimuth (anti-parallel to the direction of the wind) and local time, respectively.

A distinct pattern of wind direction depending on the radar and HF channel can be observed in Fig. 3. To see whether the diurnal and seasonal variability of the wind reflects the MSTID pattern, we imposed the contours that show the three levels of the MSTID direction occurrence rate presented in Fig. 2. Blue, red, and magenta curves represent constant occurrence rates of 3, 5, and $10 \%$, respectively, in Fig. 3a, b, and 4, 6, and $9 \%$, respectively, in Fig. 3c. It is clear from Fig. 3 that almost all contours of the enhanced occurrence rate of MSTID direction qualitatively agree well with the wind "tracks" of enhanced occurrence (they overlap or lay very closely to the corresponding wind tracks). The largest difference is related to the nighttime southwestward MSTIDs, which have no corresponding wind tracks (see explanation below). Significant differences can be also found during the winter daytime for Hok-E and Hok-F (MSTID "spots" lay above or below the wind tracks) and for the morning hours for Ekb-F (MSTID spots are displaced into greater local times). The size of some "spots" 
and the relation between wind and MSTID occurrence rate values do not agree well. Except for the nighttime southwestward MSTID spot for Hok-F and Ekb-F, the mentioned differences might be associated with the accuracy of the models used for wind pattern calculation. In addition, the wind pattern here was calculated at a single height for a certain local time, but the AGW actually propagates within an altitude range, which should be taken into account for a more accurate comparison.

The nighttime mid-latitude MSTIDs, which are observed to propagate southwestward, have been reported by many other researches (e.g., Shiokawa et al. 2003a; Otsuka et al. 2004; Kotake et al. 2007; Shiokawa et al. 2009; Otsuka et al. 2011; Ichihara et al. 2013). Shiokawa et al. (2003a) presented a statistical study of these disturbances using the data from 630-nm airglow images obtained at Shigaraki and Rikubetsu during 1998-2000. Using airglow images and DMSP satellite data, it was established that these MSTIDs at mid-latitudes are accompanied by an oscillating electric field (Shiokawa et al. 2003b). Otsuka et al. (2004) conducted geomagnetic conjugate observations of $630 \mathrm{~nm}$ airglow at Sata, Japan, and Darwin, Australia, with two all-sky CCD imagers, and found the airglow perturbations caused by MSTIDs, which were mirrored in the northern and southern hemispheres connected by geomagnetic field lines. They proposed that the polarization electric fields play an important role in the generation of the MSTIDs. There are also statistical studies by Kotake et al. (2007) and Otsuka et al. (2011) utilizing two-dimensional GPS TEC maps, which also clearly revealed such nighttime disturbances. Ichihara et al. (2013) analyzed the nighttime MSTIDs observed by the Hokkaido East HF radar from January 2007 to July 2009. The authors reported that such disturbances propagate mainly southwestward, but occasionally they propagate northeastward. Comparing the radar data and GEONET TEC data, they found that the propagation direction depends on the latitude and local time. Discussing the characteristics of the nighttime mid-latitude southwestward MSTIDs, the researchers have concluded that they cannot be explained by the AGW theory (e.g., Shiokawa et al. 2003a; Shiokawa et al. 2009; Otsuka et al. 2011). The origin of these MSTIDs is commonly attributed to the Perkins instability, which is the only known mechanism that can produce northwest-southeast stretched plasma density structures in the $\mathrm{F}$ region (Perkins 1973). The issue here is that the linear growth rate of Perkins instability is very low. However, it was shown that the electrodynamic coupling processes between the $\mathrm{E}$ and $\mathrm{F}$ layers may lead to faster development of instability (Tsunoda 2006). Recently performed three-dimensional numerical modeling, which includes the E-F coupling process with dipole magnetic field lines, reproduces the main features of the nighttime mid-latitude MSTIDs (Yokoyama and Hysell 2010; Yokoyama 2013).

In Figs. 2 and 3, the MSTID occurrence rate represents a number of localized spots distributed over the azimuth-local time plane. On the contrary, the azimuth pattern calculated from HWM07 consists of relatively smooth tracks extended in local time. In other words, there are some kinds of "gaps" in the MSTID azimuthal pattern. The gaps on Hok-F and Ekb-F pattern are located near the exact north, south, east, and west directions. Further research is needed for explanation of the gaps.

It is interesting that during the summer daytime, most disturbances observed by the Hokkaido radar at the heights of $\mathrm{E}$ and $\mathrm{F} 2$ layers propagate in exactly opposite directions, $\sim 300^{\circ}$ and $\sim 120^{\circ}$, respectively, as seen in Fig. 2a, b. Vadas (2007) presented a bulk of numerical simulation results of the GW propagation and dissipation in the thermosphere and showed that GWs with small horizontal wavelengths cannot propagate to higher altitudes in the thermosphere. Their dissipation/breaking in the lower thermosphere due to kinematic viscosity and thermal diffusivity causes thermospheric body forces, which could be a significant source of additional "secondary" GWs. These secondary waves may have larger spatial scales and longer periods and can penetrate to higher altitudes in the thermosphere and induce MSTIDs (Vadas and Fritts 2006). Therefore, most summer daytime MSTIDs observed in Hok-F might be caused by secondary GWs having their sources in the lower thermosphere. Apparently, a fraction of Hok-E summer daytime GWs might still reach the F2 layer heights. This fraction is probably responsible for the appearing of a spot of enhanced MSTID occurrence in Hok-F with azimuths close to $\sim 300^{\circ}$ at about $20 \mathrm{LT}$. The time delay between the considered spots is about $6 \mathrm{~h}$ that is quite enough for the GWs to propagate upward from the E layer to the F2 layer height. This suggestion could be verified thoroughly in a future study.

\section{Diurnal, solar cycle, and geomagnetic activity dependence of other MSTID parameters}

Figure 4 shows color-coded diurnal variation in the occurrence rate for the horizontal phase velocity (a), median period (b), horizontal wavelength (c), and relative amplitude (d). The plots contain MSTID characteristics for the entire observational period 2007-2014 (all seasons, different levels of solar activity-from minimum to maximum). Therefore, the spread of the distributions is relatively large. The most frequent daytime velocities are as follows: $60-100 \mathrm{~m} / \mathrm{s}$ for Hok-E, $110-180 \mathrm{~m} / \mathrm{s}$ for Hok-F, and 30-90 m/s for Ekb-F (see also Figs. 5 and 6 where the mode value and spread of the distributions are shown). The most frequent daytime wavelengths are 


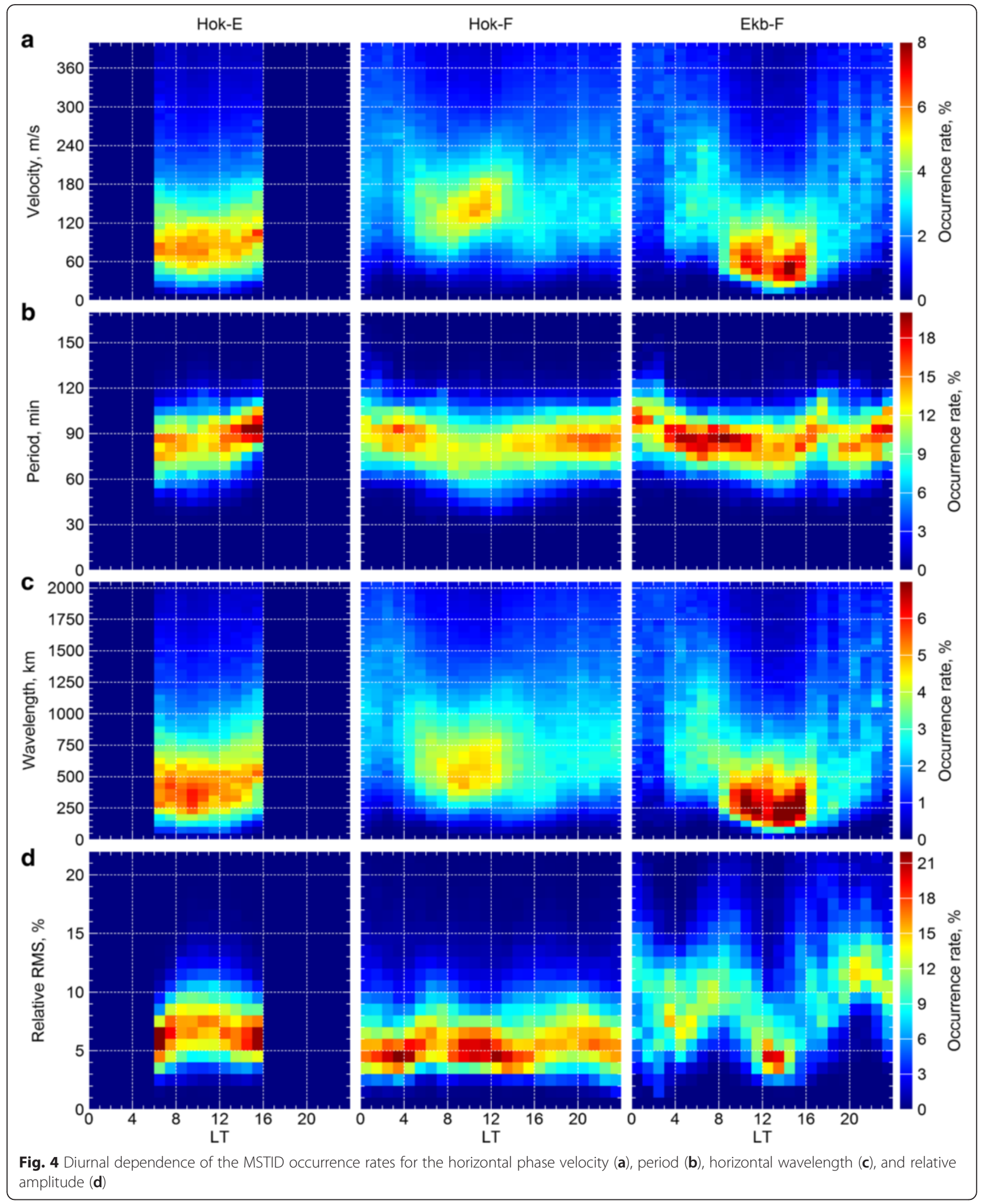

as follows: $300-550 \mathrm{~km}$ for Hok-E, $450-800 \mathrm{~km}$ for Hok-F, and 150-400 km for Ekb-F. The most frequent period is in the range of $60-100 \mathrm{~min}$.
Nighttime values of velocity, period, and wavelength for the F2 region are somewhat greater than those during the daytime. This is consistent with results of 

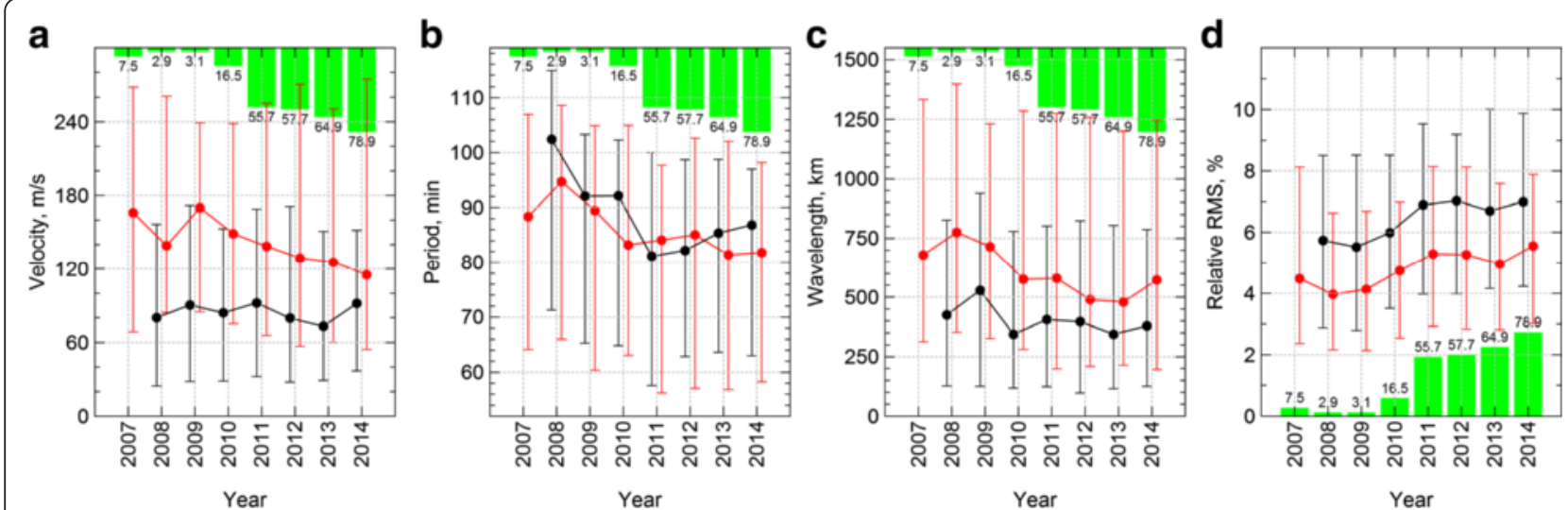

Fig. 5 Sample mode and FWHM of the MSTID horizontal phase velocity (a), period (b), horizontal wavelength (c), and relative amplitude (d) in each year of observations. Sample mode is shown by circles. Length of vertical bars indicates FWHM from its lower to upper bounds. Black and red curves correspond to the Hokkaido E and F regions, respectively. Green bars show the yearly mean sunspot number

Waldock and Jones (1986) and can be related with the increased HF reflection height during the nighttime in comparison with daytime. Velocity of GW propagating upward is expected to increase with altitude to compensate for the decreasing atmospheric density (Hines 1960). On the other hand, GWs with longer wavelength/ period are less attenuated and can propagate for longer times and reach greater heights before their dissipation (Vadas 2007). As one can see in Fig. 4 (left column), there is the same local time tendency for Hok-E, but the parameters increase from the daytime minima to the evening/morning hours. The obtained velocities and wavelengths of Hok-E are less than those of Hok-F approximately by a factor of 2 .

As seen in Fig. 4d, the relative amplitude varies within the range of 3-8\% for Hok-F; it reaches a minimum near noon and maximums at about 6 and 20 LT. In case of Ekb-F, the amplitude has a similar behavior, but varies within a greater range, from $\sim 4 \%$ near noon to $\sim 13 \%$ at 8 and 22 LT. For Hok-E, the amplitude is somewhat greater and varies within the range of 4-9\%. Similar diurnal behavior of the MSTID relative amplitude was reported by Afraimovich et al. (2008). The authors showed that the relative amplitude of TEC variations at night significantly exceeds that of the daytime; in addition, the relative amplitude is a few times greater at higher latitudes as compared to mid- and low-latitudes. Ratovsky et al. (2015) analyzed the vertical sounding data at Norilsk and Irkutsk and found that the relative variability of the maximum electron density caused by the TIDs activity is somewhat larger at the high-latitude station as compared to that in the mid-latitude, at least during winter and equinox. Thus, our results are consistent with the findings of other researchers.

To determine the solar cycle effect, we have collected all the calculated values for the Hokkaido radar into samples corresponding to different years of observation. As clearly seen in Fig. 4, the occurrence distributions of
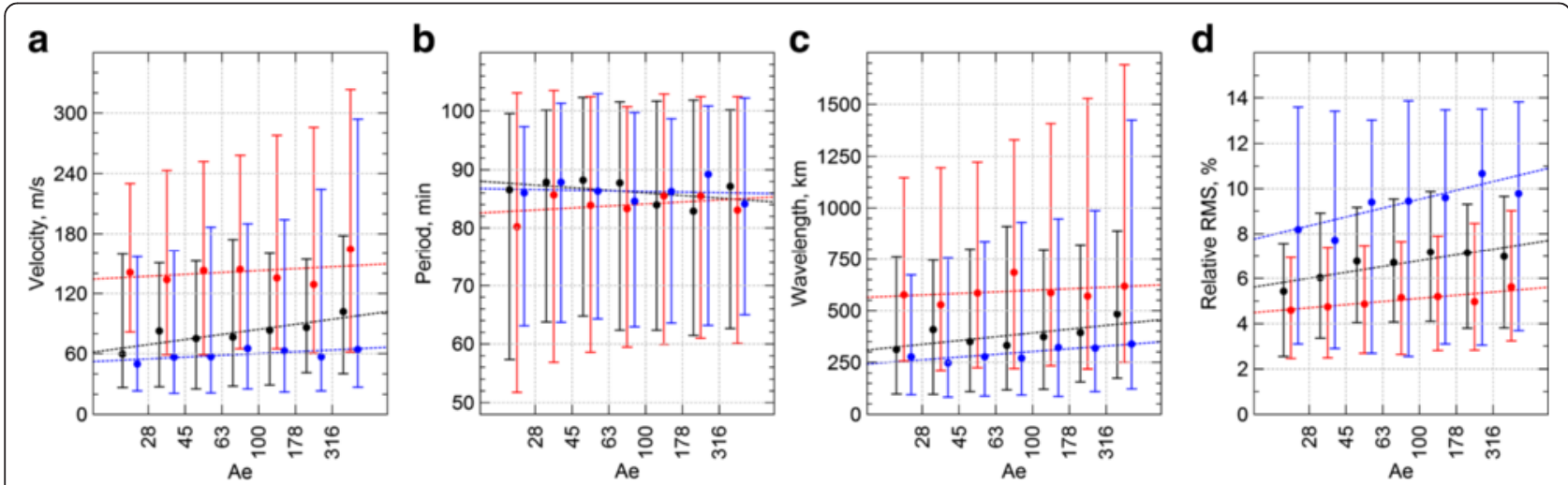

Fig. 6 Sample mode and FWHM of the MSTID horizontal phase velocity (a), period (b), horizontal wavelength (c), and relative amplitude (d) in each AE-bin. Sample mode is shown by circles. The length of vertical bars indicates the FWHM from its lower to upper bounds. The dashed lines are linear best fits for the sample mode. Black, red, and blue curves correspond to the Hokkaido E and F region and the Ekaterinburg F region, respectively 
the MSTID parameters are generally not symmetrical (not Gaussian-like). Therefore, we have characterized each sample by its mode (at the maximum occurrence rate), and the two values at which the occurrence rate is equal to half of its maximum. The difference between the two last values is the full width at half maximum (FWHM), which characterizes spread of the distribution. In Fig. 5, the circles and vertical bars show the mode values and FWHM, respectively, in each year. Black and red colors correspond to Hok-E and Hok-F, respectively. The green bars with labels show the yearly mean sunspot number obtained from the National Geophysical Data Center in Boulder (http://www.ngdc.noaa.gov/stp/ stp.html). Note that in Fig. 5a-c, the bars are reversed to aid convenient comparison. A distinct solar cycle dependence of the MSTID azimuth was not found; thus, Fig. 5 shows only the dependencies for velocity (a), period (b), wavelength (c), and relative amplitude (d). There is an obvious solar activity relationship at least for the wavelength, period, and relative amplitude in Hok-F, and for the period and relative amplitude in Hok-E. The Hok-F wavelength tends to decrease with increasing solar activity from $\sim 750 \mathrm{~km}$ in 2008 to $\sim 550 \mathrm{~km}$ in 2014. Its FWHM is relatively constant through the years $(\sim 1000 \mathrm{~km})$ and repeats the variation of the mode value. There is also a decreasing trend for the Hok-E wavelength mode from $500 \mathrm{~km}$ in 2008 to $400 \mathrm{~km}$ in 2014, but the corresponding FWHM is unstable. The relative amplitude tends to increase with increasing solar activity from 4 to $5.5 \%$ for Hok-F and from 5.5 to 7 \% for HokE from 2008 to 2014, respectively. The Hok-F period decreased with increasing solar activity from $95 \mathrm{~min}$ in 2008 to $82 \mathrm{~min}$ in 2014. The Hok-E period also decreased from $102 \mathrm{~min}$ in 2008 to $82 \mathrm{~min}$ in 2011; but after that, a weak growth to $87 \mathrm{~min}$ was observed in 2014. There is also a downward trend with increasing solar activity for the Hok-F velocity. However, the lower and upper bounds of the velocity FWHM varied in opposite manner.

The revealed trends agree with the results of other researchers. Fukushima et al. (2012) studied the nighttime MSTIDs over 7 years, from 2002 to 2009, using a 630-nm airglow imager at Kototabang, Indonesia. The average horizontal wavelength (velocity) of the MSTIDs was found to increase with decreasing solar activity. However, the authors did not find a clear dependence for the MSTID period. In addition, the MSTID occurrence rate was found to decrease with decreasing solar activity. Klausner et al. (2009) studied the MSTIDs using vertical ionosphere sounding observations at Sao Jose dos Campos, Brazil, for years of high (HSA, September 2000 to August 2001) and low solar activity (LSA, January 2006 to December 2006). From Fig. 4 in their study, the most frequent MSTID periods can be observed to be lower for HSA ( 20-60 min) than those for LSA ( 60-100 $\mathrm{min})$. They also found that the large-amplitude TIDs (virtual height greater than $60 \mathrm{~km}$ ) are present normally only during HSA. Lastovicka (2001) analyzed long radio wave absorption data for 5.5 years in the lower ionosphere obtained from the oblique ionosphere sounding by continuous waves at $270 \mathrm{kHz}$ at the middle latitude station at Pruhonice. $\mathrm{He}$ found that the relative amplitude of GW in the $E$ layer varies within the range of $2-8 \%$ and decreases from the solar activity maximum to the solar activity minimum by about $1 / 3$.

We should note that the true height of the HF reflection increases on average with increasing solar activity (due to the general lifting of the F2 layer). The true height varies from $\sim 170-270 \mathrm{~km}$ in 2008 to $\sim 190-330 \mathrm{~km}$ in 2014 , as predicted by Hok-F GBM. Hence, the solar cycle trends of the wavelength, velocity, and period were expected to be inversed as compared to the observed ones (because of decrease in the atmospheric density with height). On the other hand, as shown by numerical simulation (Vadas and Fritts 2006; Vadas 2007; Fritts and Vadas 2008), the GW dissipation height should increase with increasing solar activity. The latter is due to the increasing temperature of the thermosphere and corresponding less rapid increase in the kinematic viscosity with height. In addition, the GWs with larger horizontal wavelengths were found to dissipate at higher altitudes than those with smaller horizontal wavelengths. Based on Vadas (2007), Fukushima et al. (2012) suggested that during the solar minimum, only the GW with larger horizontal wavelengths would propagate in the thermosphere, and this might explain the fact that the observed average horizontal wavelength becomes larger with decreasing solar activity. During the active solar conditions, GWs with both small and large horizontal wavelengths are observed so that the average wavelength would become relatively small.

It is known from the earlier statistical studies of largescale TIDs (LSTIDs) that their occurrence rate clearly grows with increasing geomagnetic activity (e.g., Tsugawa et al. 2004; Ding et al. 2008). Medvedev et al. (2013) analyzed the TID population that includes the medium- and large-scale disturbances observed by the Irkutsk incoherent scatter radar coupled with the conventional vertical ionosonde data for a period from 2004 to 2009. They found that the wave activity level tends to increase with both solar and geomagnetic activity. There are also a number of papers on the geomagnetic activity effect on the MSTID parameters. Waldock and Jones (1986) compared the mean number of MSTIDs per hour and maximum daily Doppler shift with mean Ap and auroral electrojet (AE) indices. They did not find a clear correlation between the MSTID parameters and geomagnetic indices, and suggested that these waves are not caused by high-latitude generation mechanisms. Tsugawa et al. 
(2007) analyzed the MSTID activity variation revealed by the TEC observations using a dense GPS receiver network over Japan, and did not find obvious relation of the dayto-day MSTID activity variation to the geomagnetic activity. Grocott et al. (2013) investigated a relationship between solar wind-magnetosphere-ionosphere (SW-M-I) coupling and MSTID occurrence based on the GB data of the Falkland Islands SuperDARN radar (FIR) between May 2010 and April 2011. They found weak correlations for the MSTID occurrence rate with the symmetric portion of the horizontal component magnetic field near the equator (Sym-H index) and with the dayside reconnection voltage. Their results suggest a degree of association between the MSTID occurrence and the level of SW-M-I coupling, and could provide an evidence for a ducted mode of the AGW propagation from a high-latitude source to mid-latitudes. Frissell et al. (2014) analyzed the MSTID observations by the Blackstone SuperDARN radar and concluded that the AE index showed a correlation with the MSTID statistics.

To investigate the possible geomagnetic activity effect, we collected all the calculated values into samples corresponding to different intervals (bins) of the $\mathrm{AE}$ index. The $\mathrm{AE}$ index values used in this study were obtained from the World Data Center for Geomagnetism in Kyoto (http://wdc.kugi.kyoto-u.ac.jp). The samples have approximately equal sizes ( 180 days for Hok-F and $\sim 60$ days for Hok-E and Ekb-F). The sampling has been performed taking into account some time shift needed for the effect of auroral electrojet variations (originating at $\sim 70^{\circ} \mathrm{N}$ ) to appear at the radar registration region. We used the time shift of 4 and $1 \mathrm{~h}$ for the Hokkaido radar and Ekaterinburg radar, respectively. In Fig. 6, the circles and vertical bars show the mode values and FWHM, respectively, in each AE-bin. Black, red, and blue colors correspond to Hok-E, Hok-F, and Ekb-F, respectively. Figure 6 shows variations in the velocity (a), period (b), wavelength (c), and relative amplitude (d). For the MSTID azimuth, we did not find a distinct dependence on the geomagnetic activity.

Evidently, the mode values of velocity, wavelength, and amplitude have a tendency to increase with increasing $\mathrm{AE}$ index. For better illustration of the trends, we have also plotted a linear best fit by dashed lines. The clearest growth is seen for the amplitude (see Fig. 6d). Notice that the growth rate of amplitude for Ekb-F is greater than for Hok-E and Hok-F. This agrees with Afraimovich et al. (2008), who found that on average, the relative amplitude of TEC variations varies proportionally to Kp geomagnetic index, and this dependence is distinct at high-latitudes, weaker at mid-latitudes, and weakest at the equator. The upward trends in the F region for velocity and wavelength are weaker than those for the amplitude. However, as one can see from Fig. 6a, c, there is a clear increase of the upper bound and FWHM with an increasing $\mathrm{AE}$ index, at least for Hok-F and Ekb-F. One might expect that the velocity, wavelength, and period should increase with increasing geomagnetic activity just because LSTIDs start to occur relatively more often. However, this is in contrast with Fig. 6b where there is no distinct increase of the period or even of the corresponding FWHM.

Although many authors have studied the MSTID phenomena, the effect of geomagnetic activity is still unclear. Our results show that as a response to an increasing $\mathrm{AE}$ index, a relative number of the MSTIDs with higher amplitudes, velocities, and longer wavelengths increases during observations by both radars. This effect manifests more strongly for Ekb-F. A possible way of explanation is as follows. Increasing the geomagnetic activity leads to the magnetospheric energy deposition at high-latitudes. This causes the generation of additional AGWs of different scales. However, only AGW with relatively large wavelengths could propagate at long distances and reach the mid-latitudes (Vadas 2007). On the other hand, geomagnetic storms lead specifically to a global heating of the thermosphere (Emery et al. 1999), and consequently, change the neutral wind conditions (Emmert et al. 2008). AGW propagation and dissipation conditions would change accordingly (Vadas and Fritts 2006; Vadas 2007). Certainly, a significance of the effects is higher at high-latitudes and decreases toward the equator. Further investigation is needed to clarify the details here.

\section{Conclusion}

This paper is a continuation of our previous study (Oinats et al. 2015). We have significantly improved our technique for MSTID analysis based on the HF GB data. We have utilized the elevation angle information for the Hokkaido East radar, which has allowed us to map the echo regions more accurately and to distinguish the echoes reflected in the $\mathrm{E}$ and $\mathrm{F}$ ionospheric regions. The latter makes it possible to study the MSTIDs in these regions separately.

We processed the multi-year datasets of two midlatitude HF radars: the Hokkaido East radar from 2007 to 2014 and Ekaterinburg radar from 2013 to 2014. Comparison of the revealed statistical characteristics showed a high similarity between the diurnal and seasonal MSTID occurrence patterns as observed by the two radars. There are four dominant MSTID propagation directions at the F2 layer heights, with a distinct diurnal and seasonal dependence: morning northeastward (from $20^{\circ}$ to $50^{\circ}$ ), daytime southeastward (from $100^{\circ}$ to $140^{\circ}$ ), nighttime southwestward (from $190^{\circ}$ to $220^{\circ}$ ), and evening northwestward (from $280^{\circ}$ to $310^{\circ}$ ). For the $\mathrm{E}$ region, there are also northwestward disturbances 
(azimuth varies from $280^{\circ}$ to $320^{\circ}$ ), which are typical of summer daytime. Comparison with neutral wind occurrence pattern calculated using HWM07 has revealed that the dominant MSTID propagation directions match well with the anti-wind direction at least during the sunlight hours. This indicates that the observed MSTIDs are mostly caused by AGWs propagating in the thermosphere (except for the nighttime southwestward MSTIDs). The values and diurnal behavior of the MSTID horizontal velocity, wavelength, period, and relative amplitude agree well with the results published earlier, and are consistent with the properties of the GWs propagating at corresponding heights. The processing of the extensive radar datasets allowed us to study the solar cycle and geomagnetic activity dependencies of the revealed characteristics. The MSTID wavelength and period tend to decrease with an increase in solar activity. The relative amplitude increases with an increase in solar activity. The relative amplitude, wavelength, and velocity tend to increase with increasing $\mathrm{AE}$ index.

\section{Abbreviations \\ AGWs: atmospheric gravity waves; HF: high frequency; HWM07: horizontal wind model; IRI: international reference ionosphere; LT: local time; MSTID: medium-scale traveling ionospheric disturbance; SuperDARN: super dual auroral radar network.}

\section{Competing interests}

The authors declare that they have no competing interests.

\section{Authors' contributions}

AO elaborated the technique of the TID parameter estimation, provided the processing and calculations, and drafted the manuscript. NN provided the Hokkaido East SuperDARN radar data and supervised the work of AO at STEL. PP implemented the elevation angle calibration. OB provided the Ekaterinburg $\mathrm{HF}$ radar data. NN, PP, and KR participated in the discussion and interpretation of the obtained statistical results. All authors have read and approved the final manuscript.

\section{Acknowledgements}

This work was done under the financial support of the Russian Foundation for Basic Research (grants No.14-05-00259, No.14-05-00588). The work of Oinats AV was supported by the Japan Russia Youth Exchange Fellowship Program 2014. The World Data Center for Geomagnetism in Kyoto provided the AE index. The National Geophysical Data Center in Boulder provided the sunspot numbers.

\section{Author details}

'Institute of Solar-Terrestrial Physics SB RAS, 664033, Lermontov St., 126a, P.O. Box 291, Irkutsk, Russia. Institute for Space-Earth Environmental Research, Nagoya University, Furo-cho, Chikusa-ku, Nagoya 464-8601, Japan. ${ }^{3}$ University of Saskatchewan, Saskatoon, SK, Canada. ${ }^{4}$ Solar-Terrestrial Environment Laboratory (now-Institute for Space-Earth Environmental Research), Nagoya University, Nagoya, Japan.

Received: 31 March 2015 Accepted: 15 January 2016

Published online: 21 January 2016

\section{References}

Afraimovich EL, Boitman ON, Zhovty El, Kalikhman AD, Pirog TG (1999) Dynamics and anisotropy of traveling ionospheric disturbances as deduced from transionospheric sounding data. Rad Sci 34:477-487

Afraimovich EL, Lipko W, Vugmeister B (2000) Determining dynamic parameters of different-scale ionospheric irregularities over northern Siberia. J Atmos Sol Terr Phys 62:133-140
Afraimovich EL, Perevalova NP, Zhivetiev IV (2008) Relative amplitude of the total electron content variations depending on geomagnetic activity. Adv Space Res 42:1231-1237

Arnold NF, Jones T, Robinson T (1998) Validation of the CUTLASS HF radar gravity wave observing capability using EISCAT CP-1 data. Ann Geophys 16:1392-1399

Bilitza D, Altadill D, Zhang Y, Mertens C, Truhlik V, Richards P, McKinnell L-A, Reinisch BW (2014) The International Reference lonosphere 2012 - a model of international collaboration. J Space Weather Space Clim 4(A07):1-12. doi: $10.1051 /$ swsc/2014004

Blanchard GT, Sundeen S, Baker KB (2009) Probabilistic identification of high-frequency radar backscatter from the ground and ionosphere based on spectral characteristics. Rad Sci 44:RS5012. doi:10.1029/2009RS004141

Bland EC, McDonald AJ, de Larquier S, Devlin JC (2014) Determination of ionospheric parameters in real time using SuperDARN HF Radars. J Geophys Res 119(7):5830-5846

Davies K (1990) lonospheric radio. Peter Peregrinus Ltd, London

Ding F, Wan W, Liu L, Afraimovich EL, Voeykov SV, Perevalova NP (2008) A statistical study of large-scale traveling ionospheric disturbances observed by GPS TEC during major magnetic storms over the years 2003-2005. J Geophys Res 113(A00A01). doi:10.1029/2008JA013037

Drob DP, Emmert JT, Crowley G, Picone JM, Shepherd GG, Skinner W, Hays P, Niciejewski RJ, Larsen M, She CY, Meriwether JW, Hernandez G, Jarvis MJ, Sipler DP, Tepley CA, O'Brien MS, Bowman JR, Wu Q, Murayama Y, Kawamura S, Reid IM, Vincent RA (2008) An empirical model of the Earth's horizontal wind fields: HWM07. J Geophys Res 113:A12304. doi:10.1029/2008JA013668

Emery BA, Lathuillere C, Richards PG, Roble RG, Buonsanto MJ, Knipp DJ, Wilkinson P, Sipler DP, Niciejewski R (1999) Time dependent thermospheric neutral response to the 2-11 November 1993 storm period. J Atmos Sol Terr Phys 61:329-350

Emmert JT, Drob DP, Shepherd GG, Hernandez G, Jarvis MJ, Meriwether JW, Niciejewski RJ, Sipler DP, Tepley CA (2008) DWM07 global empirical model of upper thermospheric storm-induced disturbance winds. J Geophys Res 113: A11319. doi:10.1029/2008JA013541

Fritts DC, Vadas SL (2008) Gravity wave penetration into the thermosphere: sensitivity to solar cycle variations and mean winds. Ann Geophys 26:3841-3861

Frissell NA, Baker JBH, Ruohoniemi JM, Gerrard AJ, Miller ES, Marini JP, West ML, Bristow WA (2014) Climatology of medium-scale traveling ionospheric disturbances observed by the midlatitude Blackstone SuperDARN radar. J Geophys Res 119(9):7679-7697

Fukushima D, Shiokawa K, Otsuka Y, Ogawa T (2012) Observation of equatorial nighttime medium-scale traveling ionospheric disturbances in 630-nm airglow images over 7 years. J Geophys Res 117:A10324

Grocott A, Hosokawa K, Ishida T, Lester M, Milan SE, Freeman MP, Sato N, Yukimatu AS (2013) Characteristics of medium-scale traveling ionospheric disturbances observed near the Antarctic Peninsula by HF radar. J Geophys Res 118(9):5830-5841

He L-S, Dyson P, Parkinson ML, Wan W (2004) Studies of medium scale travelling ionospheric disturbances using TIGER SuperDARN radar sea echo observations. Ann Geophys 22:4077-4088

Hines (1960) Internal atmospheric gravity waves at ionospheric heights. Can J Phys 38:1441-1481

Hocke K, Schlegel K (1996) A review of atmospheric gravity waves and travelling ionospheric disturbances: 1982-1995. Ann Geophys 14:917-940

Hunsucker RD (1982) Atmospheric gravity waves generated in the high-latitude ionosphere: a review. Rev Geophys Space Phys 20(2):293-315. doi:10.1029/ RG020i002p00293

Ichihara A, Nishitani N, Ogawa T, Tsugawa T (2013) Northward-propagating nighttime medium-scale traveling ionospheric disturbances observed with SuperDARN Hokkaido HF radar and GEONET. Adv Polar Sci 24:42-49. doi:10. 3724/SP.J.1085.2013.00042

Ishida T, Hosokawa K, Shibata T, Suzuki S, Nishitani N, Ogawa T (2008) SuperDARN observations of daytime MSTIDs in the auroral and mid-latitudes: possibility of long-distance propagation. Geophys Res Lett 35:L13102. doi:10. 1029/2008GL034623

Kalikhman AD (1980) Medium-scale traveling ionospheric disturbances and thermospheric winds in the F-region. J Atmos Terr Phys 42:697-703 
Karhunen TJT, Robinson TR, Arnold NF, Lester M (2006) Determination of the parameters of travelling ionospheric disturbances in the high-latitude ionosphere using CUTLASS coherent scatter radars. J Atmos Solar-Terrestrial Phys 68:558-567. doi:10.1016/j.jastp.2005.03.021

Klausner V, Fagundes PR, Sahai Y, Wrasse CM, Pillat VG, Becker-Guedes F (2009) Observations of GW/TID oscillations in the F2 layer at low latitude during high and low solar activity, geomagnetic quiet and disturbed periods. J Geophys Res 114:A02313. doi:10.1029/2008JA013448

Kotake N, Otsuka Y, Ogawa T, Tsugawa T, Saito A (2007) Statistical study of medium-scale traveling ionospheric disturbances observed with the GPS networks in Southern California. Earth Planets Space 59:95-102

Koustov AV, Yakymenko KN, Nishitani N, Ponomarenko PV (2013) Hokkaido HF radar signatures of periodically reoccurring nighttime medium-scale traveling ionospheric disturbances detected at short ranges. J Geophys Res 119(2): 1200-1218. doi:10.1002/2013JA019422

Lastovicka J (2001) Effects of gravity and planetary waves on the lower ionosphere as obtained from radio wave absorption measurements. Phys Chem Earth C 26(6):381-386

Medvedev AV, Ratovsky KG, Tolstikov MV, Alsatkin SS, Scherbakov AA (2013) Studying of the spatial-temporal structure of wavelike ionospheric disturbances on the base of Irkutsk incoherent scatter radar and Digisonde data. J Atmos Solar-Terrestrial Phys 105-106:350-357. doi:10.1016/j.jastp.2013.09.001

Milan SE, Jones TB, Robinson TR, Thomas EC, Yeoman TK (1997) Interferometric evidence for the observation of ground backscatter originating behind the CUTLASS coherent HF radars. Ann Geophys 15:29-39

Oinats AV, Kurkin VI, Kutelev KA, Nishitani N (2012) The outlook of SuperDARN radars application for monitoring of the ionospheric dynamics in Russia. Physical Bases Instrum 1(3):3-18 (in Russian)

Oinats AV, Kurkin VI, Nishitani N, Saito A (2013) On the determination of traveling ionospheric disturbances parameters using SuperDARN radar data. Electromagn Waves Electron Syst 18(8):30-39 (in Russian)

Oinats AV, Kurkin VI, Nishitani N (2015) Statistical study of medium-scale traveling ionospheric disturbances using SuperDARN Hokkaido ground backscatter data for 2011. Earth Planets Space 67:22. doi:10.1186/s40623-015-0192-4

Oinats AV, Nishitani N, Ponomarenko P, Ratovsky K (2016) Diurnal and seasonal behavior of the Hokkaido East SuperDARN ground backscatter: simulation and observation. Earth Planets Space. doi:10.1186/s40623-015-0378-9.

Otsuka Y, Shiokawa K, Ogawa T, Wilkinson P (2004) Geomagnetic conjugate observations of medium-scale traveling ionospheric disturbances at midlatitude using all-sky airglow imagers. Geophys Res Lett 31:L15803. doi: 10.1029/2004GL020262

Otsuka Y, Kotake N, Shiokawa K, Ogawa T, Tsugawa T, Saito A (2011) Statistical study of medium-scale traveling ionospheric disturbances observed with a GPS receiver network in Japan. In: Abdu M, Pancheva D, Bhattacharyya A (eds) Aeronomy of the Earth's Atmosphere and lonosphere. Springer Netherlands, Dordrecht

Otsuka Y, Suzuki K, Nakagawa S, Nishioka M, Shiokawa K, Tsugawa T (2013) GPS observations of medium-scale traveling ionospheric disturbances over Europe. Ann Geophys 31:163-172. doi:10.5194/angeo-31-163-2013

Perkins F (1973) Spread F and ionospheric currents. J Geophys Res 78:218-226

Ponomarenko P, Nishitani N, Oinats AV, Tsuya T, St.-Maurice J-P (2015) Application of ground scatter returns for calibration of HF interferometry data. Earth Planets Space 67:138. doi:10.1186/s40623-015-0310-3

Ratovsky KG, Oinats AV and Nishitani N (2015) Comparison of polar, sub-polar and midlatitude ionospheric variability using ionosonde and SuperDARN data. In: Proceedings of Progress in electromagnetics research symposium, Prague, 6-9 July 2015

Ruppert D, Wand MP, Carroll RJ (2003) Semiparametric regression. Cambridge University Press, New York

Samson JC, Greenwald RAA, Ruohoniemi JM, Frey A, Baker KB (1990) Goose Bay radar observations of Earth-reflected, atmospheric gravity waves in the highlatitude ionosphere. J Geophys Res 95(A6):7693-7709

Shiokawa K, Ihara C, Otsuka Y, Ogawa T (2003a) Statistical study of nighttime medium-scale traveling ionospheric disturbances using midlatitude airglow images. J Geophys Res 108(A1):1052. doi:10.1029/2002JA009491

Shiokawa K, Otsuka Y, Ihara C, Ogawa T, Rich FJ (2003b) Ground and satellite observations of nighttime medium-scale traveling ionospheric disturbance at midlatitude. J Geophys Res 108(A4):1145. doi:10.1029/2002JA009639

Shiokawa K, Otsuka Y, Ogawa T (2009) Propagation characteristics of nighttime mesospheric and thermospheric waves observed by optical mesosphere thermosphere imagers at middle and low latitudes. Earth Planets Space 61(4):479-491
Stocker AJ, Arnold NF, Jones TB (2000) The synthesis of travelling ionospheric disturbance (TID) signatures in $\mathrm{HF}$ radar observations using ray tracing. Ann Geophys 18:56-64. doi:10.1007/s00585-000-0056-4

Tsugawa T, Saito A, Otsuka Y (2004) A statistical study of large-scale traveling ionospheric disturbances using the GPS network in Japan. J Geophys Res 109:A06302. doi:10.1029/2003JA010302

Tsugawa T, Kotake N, Otsuka Y, Saito A (2007) Medium-scale traveling ionospheric disturbances observed by GPS receiver network in Japan: a short review. GPS Solut 11:139-144. doi:10.1007/s10291-006-0045-5

Tsunoda RT (2006) On the coupling of layer instabilities in the nighttime midlatitude ionosphere. J Geophys Res 111:A11304. doi:10.1029/ 2006JA011630

Vadas SL, Fritts DC (2006) Influence of solar variability on gravity wave structure and dissipation in the thermosphere from tropospheric convection. J Geophys Res 111:A10S12. doi:10.1029/2005JA011510

Vadas SL (2007) Horizontal and vertical propagation and dissipation of gravity waves in the thermosphere from lower atmospheric and thermospheric sources. J Geophys Res 112:A06305. doi:10.1029/2006JA011845

Waldock JA, Jones TB (1984) The effects of neutral winds on the propagation of medium-scale atmospheric gravity waves at mid-latitudes. J Atmos Terr Phys 46(3):217-231

Waldock JA, Jones TB (1986) HF Doppler observations of medium-scale travelling ionospheric disturbances at mid-latitudes. J Atmos Terr Phys 48(3):245-260

Yokoyama T, Hysell DL (2010) A new midlatitude ionosphere electrodynamics coupling model (MIECO): latitudinal dependence and propagation of medium-scale traveling ionospheric disturbances. Geophys Res Lett 37 L08105. doi:10.1029/2010GL042598

Yokoyama T (2013) Scale dependence and frontal formation of nighttime medium-scale traveling ionospheric disturbances. Geophys Res Lett 40:4515-4519. doi:10.1002/grl.50905

\section{Submit your manuscript to a SpringerOpen ${ }^{\mathcal{O}}$ journal and benefit from:}

- Convenient online submission

- Rigorous peer review

- Immediate publication on acceptance

- Open access: articles freely available online

- High visibility within the field

- Retaining the copyright to your article

Submit your next manuscript at springeropen.com 